\title{
Tratamiento artroscópico del pinzamiento patelo- femoral lateral tras artroplastia total de rodilla primaria.
}

DOI: http//dx.doi.org/10.37315/SOTOCAV2021285569

RODRÍGUEZ COLLELL JR ${ }^{1}$, MIFSUT MIEDES $D^{1,2}$.

1. DEPARTAMENTO DE SALUD CLÍNICO-MALVARROSA DE VALÈNCIA

2. DEPARTAMENTO DE CIRUGÍA UNIVERSITAT DE VALÈNCIA

\section{Resumen.}

Las complicaciones fémoro-patelares tras una ATR a pesar de las mejoras en los diseños de los implantes y en la técnica quirúrgica siguen siendo un problema sin resolver. Dentro de esas complicaciones se encuentra el pinzamiento femoropatelar lateral, siendo motivo de revisión quirúrgica en un $0,33 \%$.El objetivo de este trabajo es presentar un caso clínico de pinzamiento fémoro-patelar tras prótesis total de rodilla, tratado mediante una facectomia lateral artroscópica.

Palabras clave: Lateral patellofemoral impingement, patella complications, total knee prosthesis, knee arthroplasty complications

\section{Summary.}

Femoro-patellar complications after TKA despite improvements in implant designs and surgical technique remain an unsolved problem. Among these complications is lateral femoro-patellar impingement, being the reason for surgical revision in $0.33 \%$. The objective of this work is to present a clinical case of femoro-patellar impingement after total knee replacement, treated by facectomy arthroscopic lateral.

\section{Correspondencia:}

Correspondencia:

Juan Ramón Rodríguez Collell

rocojura@hotmail.com 


\section{Introducción}

La articulación fémoro-patelar siempre ha sido una fuente de complicaciones tras una artroplastia total de rodilla, llegando a ser motivo de revisión quirúrgica de la prótesis, hasta en un $50 \%$ de las ocasiones ${ }^{1-9}$. Para solucionar este problema se han introducido cambios tanto a nivel técnico como en los diseños de los implantes, con el fin de mejorar la cinemática fémoro-patelar ${ }^{10-16}$, pero a pesar de estos esfuerzos, el problema persiste.

Clásicamente se ha medializado el componente patelar o lateralizado el femoral, con la finalidad de conseguir un mejor "tracking" fémoro-patelar ${ }^{10,16}$, pero al medializar el componente patelar, queda descubierta la cara lateral de la patela, provocando en ocasiones un pinzamiento lateral; éste contacto anormal, sobre todo en flexión, del componente patelar con el del fémur puede provocar dolor. Se trata de una complicación poco frecuente, aunque sí referida en la literatura.

El objetivo de este trabajo es presentar un caso clínico de pinzamiento fémoro-patelar tras prótesis total de rodilla, tratado mediante una facectomia lateral artroscópica.

\section{Caso Clínico}

Mujer de 84 años de edad que fue intervenida de su rodilla derecha implantándose una artroplastia total de rodilla "Attune" PS cementada tricompartimental. Desde el inicio, la mujer refería dolor en la cara anterior de la rodilla. La rodilla era estable y presentaba un rango de movilidad de $0^{\circ}$ de extensión a $120^{\circ}$ de flexión.

A la exploración el dolor se localizaba en el borde lateral de la rótula y aumentaba a la palpación. El alerón lateral rotuliano estaba tenso y permitía un desplazamiento medial de la rótula inferior al $25 \%$ de la anchura rotuliana en $20^{\circ}$ de flexión.

En la $\mathrm{Rx}$ axial de rótula a $45^{\circ}$ se observaba una prominencia ósea lateral al borde del botón rotuliano (Fig. 1).

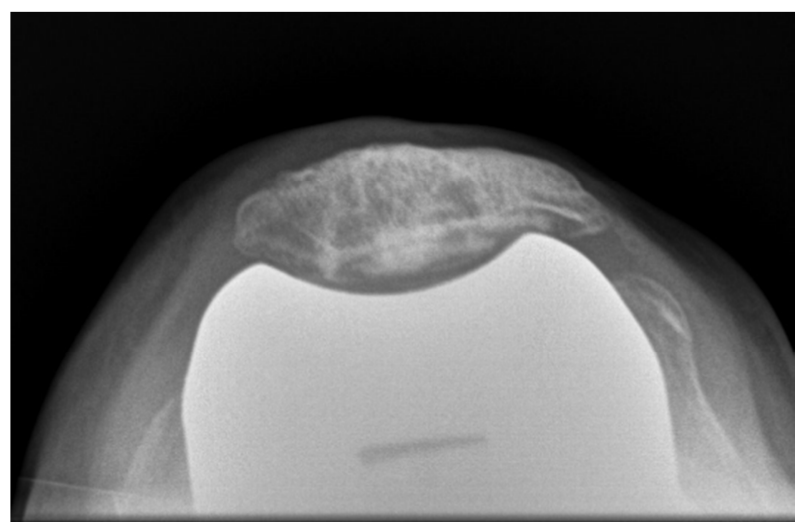

Figura 1: Imagen de radiografía axial, donde se observa el pinzamiento lateral externo del borde patelar con el componente femoral.
Ante la falta de mejoría de la paciente con tratamiento rehabilitador, se practicó una artroscopia de la rodilla a través de los dos portales estándar antero-lateral y anteromedial y un tercer portal accesorio supero-externo. A través del portal supero-externo con un condrotomo de $4,5 \mathrm{~mm}$ se resecó la prominencia ósea lateral al botón rotuliano (Figs. $2,3,4,5)$.

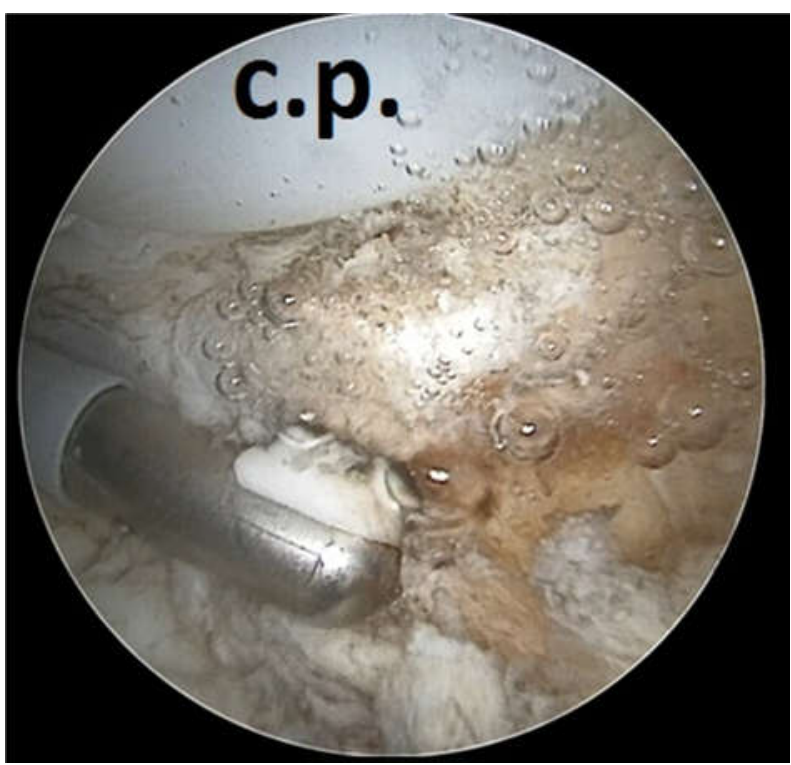

Figura 2: Imágen artroscópica donde se puede apreciar el botón rotuliano, o componente patelar (c.p.), y el reborde óseo de la cara lateral de la patela.

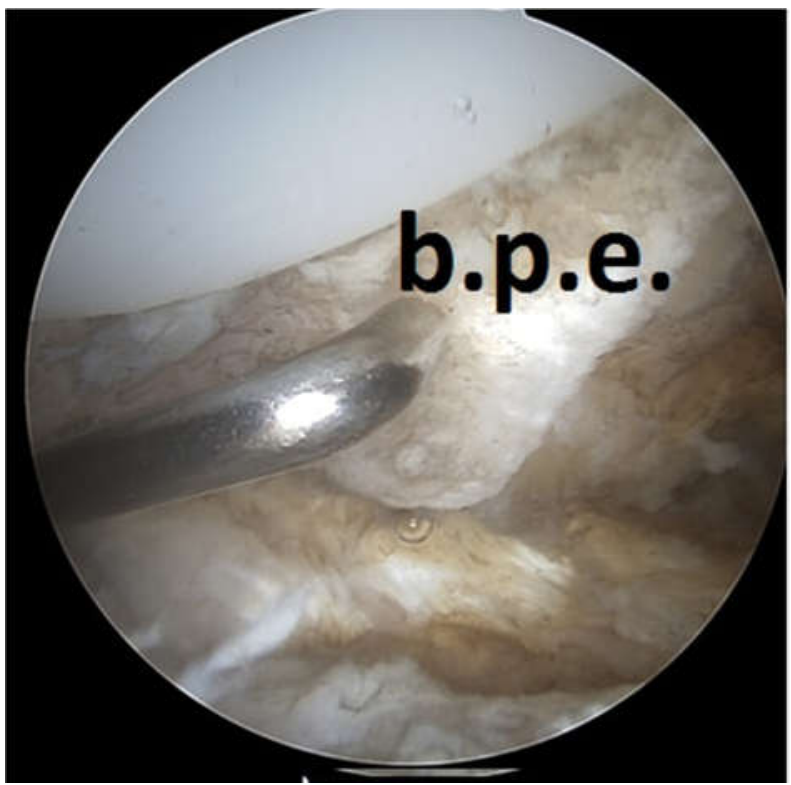

Figura 3: Imágen artroscópica donde se puede apreciar el el reborde óseo de la cara lateral de la patela, tras desbridamiento con el vaporizador. 


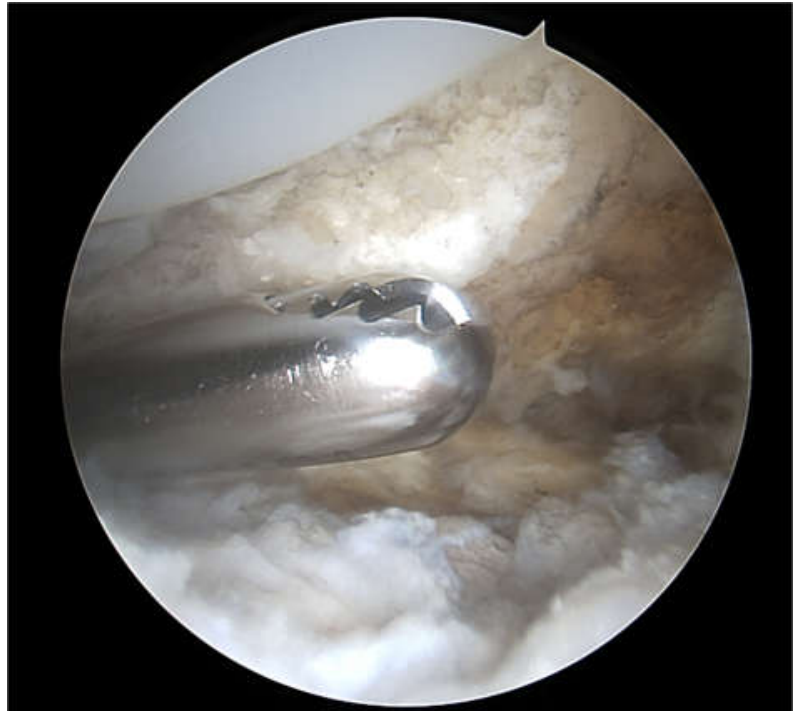

Figura 4: Imágen artroscópica donde se observa la resección del reborde óseo de la cara lateral de la patela desde el portal súpero-externo.

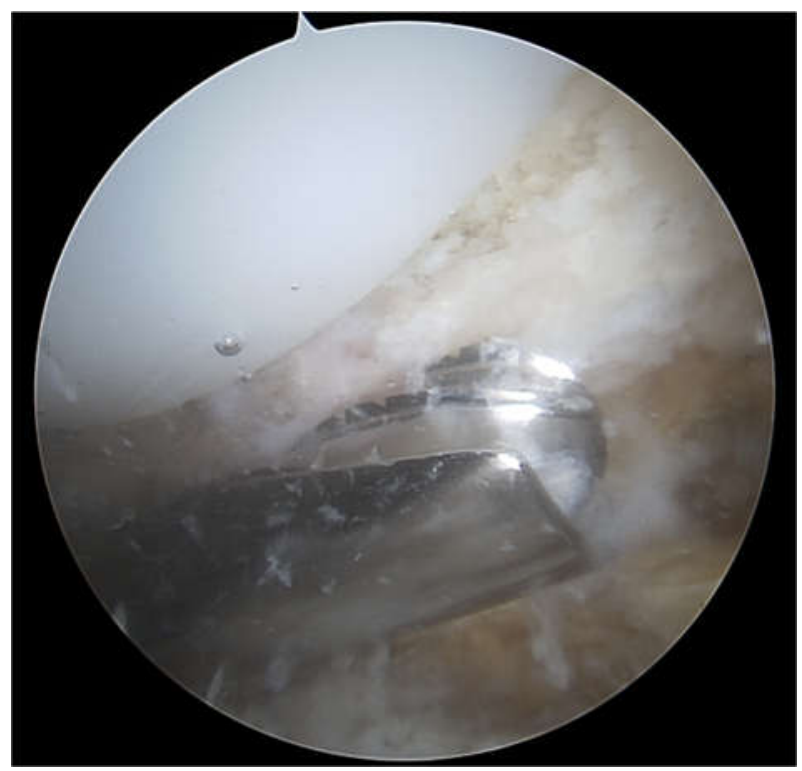

Figura 5: Imágen artroscópica donde se observa la resección del reborde óseo de la cara lateral de la patela.

y con un vaporizador se liberó el alerón rotuliano externo en su inserción en la rótula (Fig. 6). La disminución del dolor ya desde las primeras visitas fue muy llamativa, para desaparecer por completo al mes y medio de la cirugía quedando la paciente asintomática.

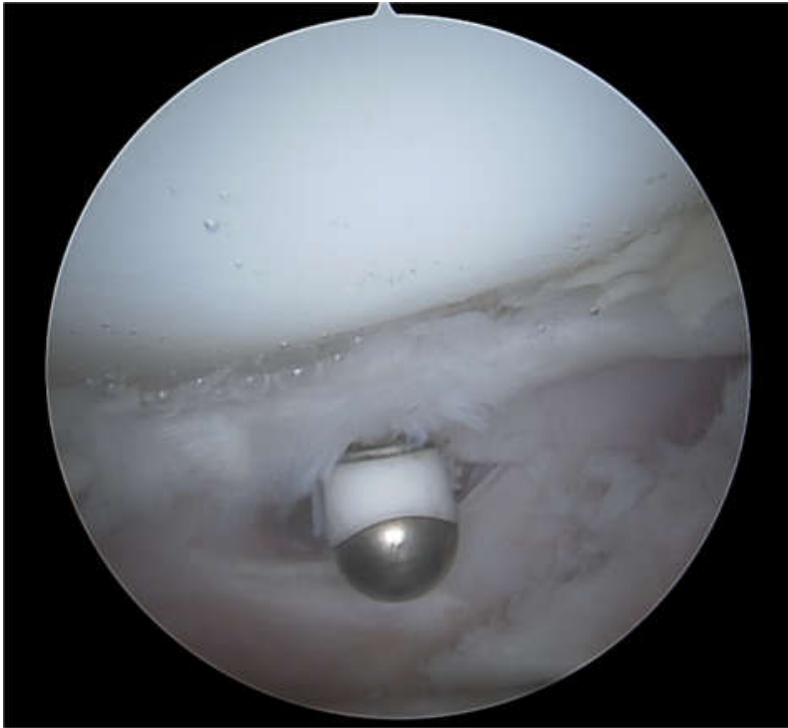

Figura 6: Imagen artroscópica de la liberación del alerón rotuliano externo.

\section{Discusión}

Las complicaciones fémoro-patelares tras una ATR a pesar de las mejoras en los diseños de los implantes y en la técnica quirúrgica siguen siendo un problema sin resolver. Dentro de esas complicaciones se encuentra el pinzamiento femoro-patelar lateral, siendo motivo de revisión quirúrgica en un $0,33 \%{ }^{17}$. Se trata de una combinación de dolor lateral o subpatelar, asociado a un contacto patelar con el implante femoral en la proyección de Merchant, aunque en numerosas ocasiones este problema se resuelve con terapia física, no requiriendo cirugía.

Nikolaus y cols. $^{17}$ describieron 9 casos de pacientes con dicho diagnóstico, descartando malrotaciones del componente femoral, tibial, o de ambos, tamaños pequeños de patela, un mal corte patelar, un mal "tracking" o la combinación de varios de estos factores.

Cercek y cols. ${ }^{18}$ publicaron una serie de 19 rodillas en 18 pacientes que requirieron revisión quirúrgica por la misma patología, con mejoría en la escala KSS estadísticamente significativa en todos los casos a las 8,16 semanas y al año de la intervención.

Ritter y cols. ${ }^{19}$ revisaron 980 ATR desde 1997 a 1998. El $46 \%$ de esas rodillas presentaban en el estudio radiográfico, contacto entre la faceta patelar lateral y el componente femoral, sin mostrar correlación significativa en los resultados de valoración de la escala KSS o en el rango de movimiento. Por ello, a los hallazgos radiográficos hay que sumar los signos y síntomas clínicos de pinzamiento rotuliano, como crepitación rotuliana, un impacto rotuliano positivo, prueba de pinzamiento lateral de la rodilla, y dolor subpatelar o anterior para confirmar un diagnóstico de pinzamiento fémoro-patelar lateral (PFPL). 
Después de revisar la literatura, en los pacientes a los que se les ha sometido a una cirugía de revisión por supuesta PFPL, y comparando las características clínicas y radiográficas entre estos pacientes y controles, está claro que esta rara la complicación existe. Sin embargo, no se ha podido identificar ningún factor de riesgo, factores radiográficos o clínicos, que ayudarían a determinar si alguien tiene un mayor riesgo de desarrollar PFPL sintomático. Por lo tanto, no podemos definir claramente la razón subyacente por la cual un grupo pequeño de estos pacientes desarrolla esta rara complicación.
Cuando un paciente tiene dolor de rodilla lateral o subpaterar y además existe evidencia de pinzamiento radiográfico, el PFPL debe ser considerado como una posible etiología de los síntomas de este paciente. Sin embargo, los resultados de la cirugía de revisión son un recordatorio aleccionador de que no todos los pacientes mejorarán con la revisión de su ATR. Por ello, se debe informar a los pacientes que presenten signos y síntomas de PFPL que el primer tratamiento debe ser conservador y pueden requerir una revisión quirúrgica en algunos casos.

\section{Bibliografía}

1. Ayers DC, Dennis DA, Johanson NA, et al. Common complications of total knee arthroplasty. J Bone Joint Surg Am 1997;79:278.

2. Brick GW, Scott RD. The patellofemoral component of total knee arthroplasty. Clin Orthop Relat Res 1988;231:163.

3. Castro Jr FP, Chimento G, Munn BG, et al. An analysis of Food and Drug Administration medical device reports relating to total joint components. J Arthroplasty 1997;12:765.

4. Healy WL, Wasilewski SA, Takei R, et al. Patellofemoral complications following total knee arthroplasty. Correlation with implant design and patient risk factors. J Arthroplasty 1995;10:197

5. Kelly MA. Patellofemoral complications following total knee arthroplasty. Instr Course Lect 2001;50:403.

6. Leopold SS, Silverton CD, Barden RM, et al. Isolated revision of the patellar component in total knee arthroplasty. J Bone Joint Surg Am 2003;85A:41.

7. Lonner JH, Lotke PA. Aseptic complications after total knee arthroplasty. J Am Acad Orthop Surg 1999;7:311.

8. Rand JA. Extensor mechanism complications after total knee arthroplasty. Instr Course Lect 2005;54:241.

9. Sharkey PF, Hozack WJ, Rothman RH, et al. Insall Award paper. Why are total knee arthroplasties failing today? Clin Orthop Relat Res 2002;404:7.

10. Anglin C, Brimacombe JM, Wilson DR, et al. Biomechanical consequences of patellar component medialization in total knee arthroplasty. J Arthroplasty 2009;28:28.

11. Barrack RL, Burak C. Patella in total knee arthroplasty. Clin Orthop Relat Res 2001;389:62.

12. Eisenhuth SA, Saleh KJ, Cui Q, et al. Patellofemoral instability after total knee arthroplasty. Clin Orthop Relat Res 2006;446:149.

13. Malo M, Vince KG. The unstable patella after total knee arthroplasty: etiology, prevention, and management. J Am Acad Orthop Surg 2003;11:364

14. Miller MC, Zhang AX, Petrella AJ, et al. The effect of component placement on knee kinetics after arthroplasty with an unconstrained prosthesis. J Orthop Res 2001;19:614.

15. Pagnano MW, Trousdale RT. Asymmetric patella resurfacing in total knee arthroplasty. Am J Knee Surg 2000;13:228.

16. Yoshii I, Whiteside LA, Anouchi YS. The effect of patellar button placement and femoral component design on patellar tracking in total knee arthroplasty. Clin Orthop Relat Res 1992;275:211.

17. Nikolaus OB, Larson DR, Hansen AD. Lateral patellar facet impingement after primary total knee arthroplasty: It does exist. . J Arthroplasty $2014 ; 29: 970-6$.

18. Cercek R, Jacovsky D, Kieffer K, et al. Lateral patellofemoral impingement: a cause of treatable pain after TKA. J Knee Surg 2011;24:181.

19. Ritter MA, Keating EM, Faris PM, et al. Lateral patellar contact after total knee arthroplasty: an analysis of the effects on postoperative pain and outcome. J Arthroplasty 2006;21:1017. 